\title{
Opportunistic Cooperative ARQ Transmission Scheme in Cellular Networks
}

\author{
Dandan Wang ${ }^{\dagger}$, Chia-Chin Chong*, Fujio Watanabe*, Hlaing Minn ${ }^{\dagger}$ and Naofal Al-Dhahir ${ }^{\dagger}$ \\ *DoCoMo Communications Laboratories USA, Inc. \\ 3240 Hillview Avenue, Palo Alto, CA 94304 \\ Email: \{cchong, watanabe\}@docomolabs-usa.com \\ ${ }^{\dagger}$ Dept. of Electrical Engineering, \\ University of Texas at Dallas, Richardson, TX 75083 \\ Email: \{dxw053000, hxm025000, aldhahir\}@utdallas.edu
}

\begin{abstract}
${ }^{1}$ Abstract-In cellular networks, a mobile station (MS) may be located in the coverage hole or out of the coverage of the base station (BS). In such a scenario, one or more relay stations (RSs) can be used to facilitate the transmission. Here, a thresholdbased opportunistic cooperative ARQ transmission scheme is proposed. Based on this scheme, the transmission between the BS and the MS can be separated into two parts: firstly, the transmission between BS and RSs (BS-RSs link) and secondly, the transmission between RSs and MS (RSs-MS link). The proposed scheme is different from the conventional ACK/NACK used for unicast transmission, in which two new types of ARQ messages are introduced for multicast transmission, namely, the relayassociated ACK/NACK (i.e., R-ACK/R-NACK) and the cooperative $A C K / N A C K$ (i.e., C-ACK/C-NACK), for BS-RSs link and RSs-MS link, respectively. A pre-defined threshold is applied to evaluate the reliability of the BS-RSs link. If the number of reliable $R S s$ is larger than the threshold value, the reliable RSs will transmit the packet to the MS in a cooperative manner. Since reliable transmission exists in the BS-RSs link, the BS will release the packet and further transmission of this packet occurs only between reliable RSs and the MS. Therefore, the proposed scheme can reduce the latency of cellular networks and the processing burden on the BS as well as achieve higher throughput as shown in the performance analysis and simulation results.
\end{abstract}

\section{INTRODUCTION}

A new form of spatial diversity, whereby diversity gains are achieved via the cooperation of users has been recently proposed using a virtual antenna system. Some of the early works have shown that node cooperation transmission can increase the sum-rate over non-cooperative transmission for ergodic fading links [1]-[3]. In [4], the space-time-coded cooperative diversity protocols were developed for combating multipath fading across multiple protocol layers in a wireless network. Later, efficient protocols that achieve the cooperative diversity gains were proposed in [5], which include fixed-relaying schemes (e.g., amplify-and-forward and decode-and-forward) and best-relay-selection schemes (e.g., channel-measure-based relay selection and incremental relay scheme).

The Automatic Repeat reQuest (ARQ) protocol is used at the link layer as an effective means to overcome the channel

\footnotetext{
${ }^{1}$ This work was done when D. Wang was an intern in DoCoMo USA Labs.
}

fading, where cyclic redundancy check (CRC) is usually used for error check and retransmissions are requested only if the received packet is erroneous [6]. Several relay selection schemes have been reported in the literature which exploit the limited feedback from the destination terminal using ARQ. In [7], a relay selection scheme based on the generalization of Hybird-ARQ (HARQ) is proposed, in which retransmitted packets do not need to come from the original source but could instead be sent by relay nodes that overhear the transmission. Similarly, [8]-[10] proposed schemes based on ARQ/HARQ for delay-limited single-relay fading channel. However, all of the schemes reported in [7]-[10] are limited to select one relay node only. Furthermore, they also assumed that the source can at least receive the acknowledgement (ACK) or negative acknowledgement (NACK) from the destination correctly, which might not be true in practical scenarios (e.g., nontransparent mode in $802.16 \mathrm{j}$ ). A cooperative protocol design with truncated ARQ for multiple relay nodes is studied in [11], in which all relay nodes that receive both the overheard packet from the source and NACK from the destination correctly can cooperatively transmit the overheard packet to the destination. Note that this scheme is designed to utilize the feedback from the destination to the source.

In this paper, we consider a practical scenario in which the destination (mobile station (MS)) can be located at the coverage hole or out of the coverage of the source (base station (BS)). In such a scenario, the transmissions between the BS and the MS can be facilitated by one or more relay stations (RSs). In particular, a threshold-based opportunistic cooperative ARQ transmission scheme is proposed. Conventionally, ACK/NACK messages are required only in the unicast transmission. Here, two new types of ACK/NACK messages are introduced for the multicast transmission. The proposed scheme is designed to utilize the cooperative diversity gain in order to reduce the latency of the network and to improve the throughput.

The remainder of this paper is organized as follows: In Section II, an opportunistic cooperative ARQ transmission scheme is proposed, which includes the system model and 


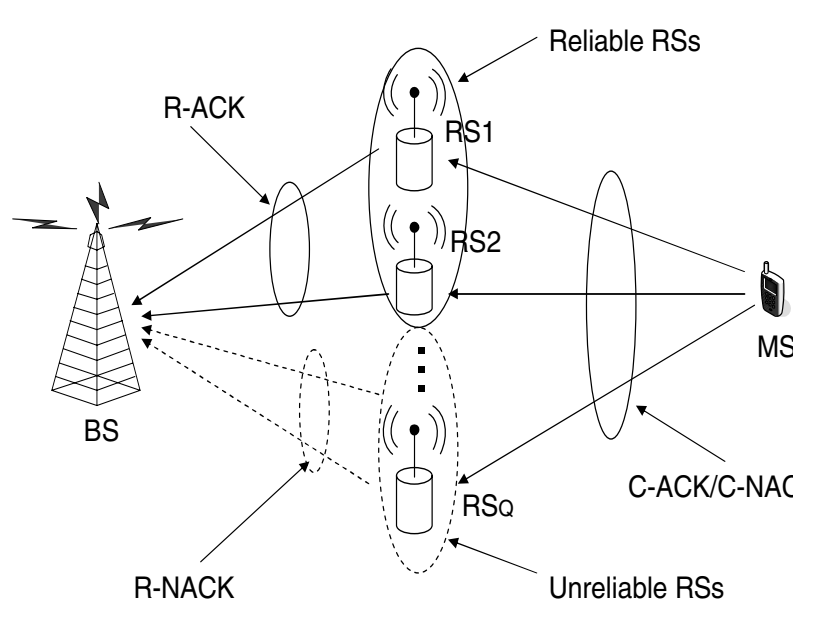

Fig. 1. The illustration of R-ACK/R-NACK and C-ACK/C-NACK.

scheme description. In Section III, the detailed throughput analysis is given. Simulation results are presented in Section IV. Finally, Section V concludes the paper.

\section{A Novel Opportunistic CoOperative ARQ TRANSMISSION SCHEME}

\section{A. System Model and Scheme Description}

We consider a time-division-duplex (TDD) cellular network consisting of a BS, a MS and $Q$ RSs, each equipped with single antenna. These $Q$ RSs have good channel power gain with the MS during the periodic ranging process. We assume that a BS/MS/RS can not transmit and receive simultaneously, and the RSs operate in a decode-and-forward fashion. The focus of our proposed scheme is on the downlink transmission which can be separated into two parts. During the first part of transmission, the BS transmits the packet to $Q$ RSs in a multicast mode with CRC attached. In the second part of transmission, the RSs transmit the packet to the MS in a cooperative manner, e.g. using distributed space-time codes. Additionally, we also introduce two new types of ARQ messages, namely, the relay-associated ACK/NACK (i.e., R-ACK/R-NACK) and the cooperative $A C K / N A C K$ (i.e., C-ACK/C-NACK). Note that these newly-defined messages are different from the conventional ACK/NACK used in the unicast mode, in which $\mathrm{R}-\mathrm{ACK} / \mathrm{R}-\mathrm{NACK}$ is the feedback message from the RSs in a multicast group to the BS, while C-ACK/C-NACK is the feedback from the MS to the $Q$ RSs as shown in Fig. 1. In this paper, we assume the R-ACK/R-NACK will not collide by using some CDMA/FDMA techniques in the transmissions.

Let the initial transmission status be defined by $m=1$, $n_{1}=1, n_{2}=1$ and $W_{R}=0$ in which $m$ is the transmission number, $n_{1}$ and $n_{2}$ are the packet numbers in the first part and second part of transmission, respectively. $W_{R}$ is the latest packet number waiting to be transmitted at the RSs. Note that there could be a queue waiting at the RSs which includes the

\begin{tabular}{|l|l|l|l|l|}
\hline $\begin{array}{c}\text { Data packet } \\
\text { from BS to RSs }\end{array}$ & $\begin{array}{l}\text { R-ACK/R-NACK } \\
\text { from RSs to BS }\end{array}$ & $\begin{array}{l}\text { Notification message } \\
\text { from BS to RSs }\end{array}$ & $\begin{array}{l}\text { Data packet } \\
\text { from RS(s) to MS }\end{array}$ & $\begin{array}{l}\text { C-ACK/C-NACK } \\
\text { from MS to all RSs }\end{array}$ \\
\hline st $^{\text {st }} \mathrm{TS} \longrightarrow$ & $2^{\text {nd }} \mathrm{TS} \longrightarrow$ \\
\hline
\end{tabular}

Fig. 2. Downlink transmission sequences.

packet number ranging from $n_{2}+1$ to $W_{R}$. We also define $L_{1}(n), L_{2}(n)$, and $L(n)=L_{1}(n)+L_{2}(n)$ as the number of transmissions used for the $n$th packet in the first part, second part, and overall transmission, respectively. Initially, we set $L\left(n_{1}\right)=L_{1}\left(n_{1}\right)=L_{2}\left(n_{1}\right)=L\left(n_{2}\right)=L_{1}\left(n_{2}\right)=$ $L_{2}\left(n_{2}\right)=0$ for all the packets. We define $v$ as a pre-defined threshold for $1 \leq v \leq Q$ and $L_{\max }$ as the maximum number of transmissions used for a packet. The downlink transmission sequences are illustrated in Fig. 2. The detailed protocol is described in the following steps associated with time slot (TS) for the $m$ th transmission:

1) 1st TS: The BS sends the $n_{1}$ th packet (with CRC attached) to all the $Q$ RSs. Thus, $L_{1}\left(n_{1}\right)=L_{1}\left(n_{1}\right)+1$.

2) 2nd TS: All the $Q$ RSs will check the CRC received from the BS and feedback R-ACK/R-NACK depending on whether they receive the packet correctly or not. The RSs which have received the $n_{1}$ th packet correctly are defined as reliable RSs (see Fig. 1) for the $n_{1}$ th packet. Let $\mathbb{Q}\left(n_{1}\right)=\left\{R S_{i}: R S_{i}\right.$ receives the $n_{1}$ th packet correctly, $i=1,2, \ldots Q$.$\} and store the n_{1}$ th packet at the reliable RSs.

3) 3rd TS: Based on the number of received R-ACKs $\left(Q_{v}\right)$, the BS will send a positive/negative notification message to the RSs. If $Q_{v} \geq v$ and $L_{1}\left(n_{1}\right) \leq L_{\max }$, then reliable communication can be established between the BS and the RSs and the BS will send a positive notification message. If $Q_{v}<v$ and $L_{1}\left(n_{1}\right)<L_{\max }$, the BS will send a negative notification message. In the special case of $L_{1}\left(n_{1}\right)=L_{\max }$ and $Q_{v}<v$, the BS will also send a positive notification message so that all the available reliable RSs can send the $n_{1}$ th packet to the MS.

4) 4th TS: If the RSs receive the positive notification message from the BS, the packet number waiting at RSs will be updated, i.e., $W_{R}=W_{R}+1$, otherwise, $W_{R}$ remains unchanged. The RSs will then check the received C-ACK/C-NACK (sent from the MS in the $(m-1)$ th transmission).

- If C-ACK is received, then $n_{2}=n_{2}+1$, which means that RSs are ready to transmit the next packet to MS.

- If $n_{2}>W_{R}$, meaning no packet to be transmitted from the RSs to the MS, RSs will have time to do other processing, e.g., help transmission in other networks.

- If $n_{2} \leq W_{R}, \mathbb{Q}\left(n_{2}\right)$ will send the $n_{2}$ th packet to the MS in a cooperative manner. Thus, $L_{2}\left(n_{2}\right)=$ $L_{2}\left(n_{2}\right)+1=1$. If $W_{R}$ is updated to be $n_{2}$ in this transmission, i.e., the reliable RSs are ready to 
send the $n_{2}$ th packet at the same transmission that they receive the positive notification message of the $n_{2}$ th packet, then $L\left(n_{2}\right)=L_{1}\left(n_{2}\right)+L_{2}\left(n_{2}\right)-$ 1. Otherwise, $L\left(n_{2}\right)=L_{1}\left(n_{2}\right)+L_{2}\left(n_{2}\right)$.

- If C-NACK is received (or neither C-NACK nor $\mathrm{C}$-ACK is receiver) and $L\left(n_{2}\right)<L_{\max }$, then $n_{2}$ remains unchanged and $\mathbb{Q}\left(n_{2}\right)$ will resend the $n_{2}$ th packet to the MS and thus, $L_{2}\left(n_{2}\right)=L_{2}\left(n_{2}\right)+1$ and $L\left(n_{2}\right)=L\left(n_{2}\right)+1$.

For $m=1$, if the RSs receive the negative notification message from BS, RSs will not send the first packet to MS.

5) 5th TS: The MS feedbacks C-ACK/C-NACK to the RSs based on whether it receives the packet from the RSs correctly or not.

Step 1) to Step 5) will be repeated for $m=m+1$. When the reliable transmission is established between BS and RSs, the BS will release the current packet and the reliable RSs will be in charge of this packet in the second part of transmission. This clearly shows that such a scheme can reduce the processing burden on the BS and the overall latency of the network. Note that by allowing the transmission from RSs to MS only if there are sufficient number of reliable RSs (i.e. $Q_{v} \geq v$ ), higher cooperative diversity gain can be achieved at the MS. A larger threshold means a longer delay in the first part of transmission but smaller delay in the second part of transmission. Thus, different threshold values will have different impact on the overall performance which depends on the BS-RSs and RSsMS link conditions as will be shown in Section III.

\section{B. Signal and Channel Models}

As described in the previous section, we consider the scenario where the MS is out of the coverage region of the BS. Therefore, the BS and the MS are usually located far away from each other. Without loss of generality, we assume that the instantaneous channel gains of the BS-RSs link and the RSsMS link are independent and a perfect channel knowledge is assumed to be available at the receiver side (i.e., RSs/MS). In this paper, we consider flat fading channels. For frequencyselective channels, orthogonal frequency division multiplexing (OFDM) can be used to combat the frequency selectivity. The channel coefficients of different transmissions are independent from each other. For the first part of the transmission, let $x^{n}\left(u_{1}\right)$ be the $u_{1}$ th symbol in the $n$th packet sent by the BS. The equivalent baseband representation at $R S_{i}$ is given by

$$
r_{i}^{n}\left(u_{1}\right)=h_{i}^{n}\left(u_{1}\right) x^{n}\left(u_{1}\right)+w_{i}^{n}\left(u_{1}\right), \quad i=1,2, . ., Q,
$$

where $h_{i}$ is the channel gain between the BS and $R S_{i}$, and $w_{i}$ is the additive white complex Gaussian noise (AWGN) with zero mean and variance $N_{0}^{(1)}$ at $R S_{i}$.

For the second part of the transmission, let $s_{j}^{n}\left(u_{2}\right)$ denote the $u_{2}$ th symbol in the $n$th packet sent by $R S_{j}$ in $\mathbb{Q}(n)$ to the MS. The corresponding received signal at the MS is given by

$$
y^{n}\left(u_{2}\right)=\sum_{R S_{j} \in \mathbb{Q}(n)} \alpha_{j}^{n} s_{j}^{n}\left(u_{2}\right)+z^{n}\left(u_{2}\right),
$$

where $\alpha_{j}$ is the channel gain between $R S_{j}$ and the MS and $z$ is the AWGN with zero mean and variance $N_{0}^{(2)}$ at the MS.

In this paper, the following notations are used: $p_{i}$ and $q_{i}$ denote the packet error rate (PER) of the transmission from BS to $R S_{i}$, and from $R S_{i}$ to MS, respectively, and $q_{i, j}$ denotes the PER when $R S_{i}$ and $R S_{j}$ transmit to the MS in a cooperative manner. In order to demonstrate the performance of the proposed scheme, we assume all the ACK/NACK are received correctly in the analysis.

\section{Outage, Delay And Throughput Analysis}

In this section, we analyze the outage probability, delay, and throughput of the proposed scheme. We first assume the $n$th packet is sent from the BS and no packet is transmitted or waiting to be transmitted between the RSs and the MS. As described in Section II, the total number of transmissions used to transmit the $n$th packet is $L(n)=L_{1}(n)+L_{2}(n)-1$. When the BS sends a positive notification message to RSs, there are two possible events for the packet to be correctly received at the MS, and two corresponding possible events for the packet to be incorrectly received at the MS (i.e., lost packet), respectively, within $L_{\max }$ transmissions:

- Event $E_{k, l}$ : RSs receive C-ACK with the condition of: $Q_{v} \geq v, L_{1}(n)=k$ for $1 \leq k \leq L_{\max }$ and $L_{2}(n)=l$ for $1 \leq l \leq L_{\max }-k+1$. Thus $L(n)=L_{1}(n)+L_{2}(n)-1=$ $k+l-1$.

- Event $E_{L, 1}^{\prime}$ : RSs receive C-ACK with the condition of: $0<Q_{v}<v, L_{1}(n)=L_{\max }$ and $L_{2}(n)=1$. Thus, $L(n)=L_{1}(n)+L_{2}(n)-1=L_{\max }$.

- Event $E_{k}^{c}$ : RSs never receive C-ACK with the condition of: $Q_{v} \geq v, L_{1}(n)=k$ for $1 \leq k \leq L_{\max } . L(n)=$ $L_{\max }$ and $L_{2}(n)=L_{\max }-L_{1}(n)+1=L_{\max }-k+1$.

- Event $E_{L, 1}^{\prime c}$ : RSs never receive C-ACK with the condition of: $0<Q_{v}<v, L_{1}(n)=L_{\max } . L_{2}(n)=L_{\max }-$ $L_{1}(n)+1=1$.

When BS sends a negative notification message to RSs, the following event for packet loss is defined:

- Event $E_{\emptyset}$ : None of the RSs receive the packets correctly within $L_{\max }$ transmissions.

\section{A. Outage Analysis}

We define $I_{k}(i)$ and $J_{k}(i)$ as

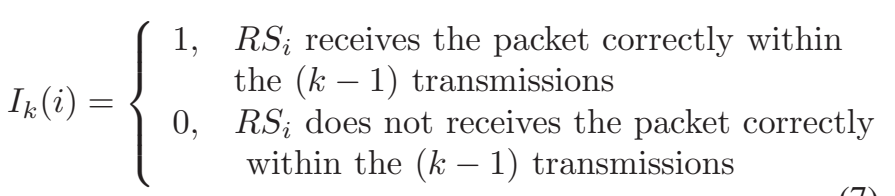

and

$J_{k}(i)= \begin{cases}1, & R S_{i} \text { receives the packet correctly } \\ \text { at the } k \text { th transmission and } I_{k}(i)=0 \\ 0, \quad \begin{array}{l}R S_{i} \text { does not receive the packet correctly } \\ \text { within } k \text { transmissions or } I_{k}(i)=1\end{array}\end{cases}$ 


$$
\begin{aligned}
& P\left(E_{k}^{c}\right)=\sum_{Q_{v}=v}^{Q} \sum_{\mathbb{D}_{Q_{v}}^{k}}\left\{\prod_{i=1}^{Q}\left[p_{i}^{k\left(1-I_{k}(i)-J_{k}(i)\right)}\left(1-I_{k}(i) p_{i}^{k-1}\right) p_{i}^{(k-1) J_{k}(i)}\left(1-J_{k}(i) p_{i}\right)\right] q_{G\left(D_{Q_{v}}^{k}\right)}^{L_{\max }+1-k}\right\}, \\
& \left.P\left(E_{L, 1}^{\prime c}\right)=\sum_{Q_{v}=1}^{v-1} \sum_{\mathbb{D}_{Q_{v}}^{k}}\left\{\prod_{i=1}^{Q}\left[p_{i}^{L_{\max }\left(1-I_{L_{\max }}(i)-J_{L_{\max }}(i)\right)}\left(1-I_{L_{\max }}(i) p_{i}^{L_{\max }-1}\right) p_{i}^{\left(L_{\max }-1\right) J_{L_{\max }}(i)}\left(1-J_{L_{\max }}(i) p_{i}\right)\right] q_{G\left(D_{Q_{v}}^{k}\right)}\right)\right\} . \\
& P\left(E_{k, l}\right)=\sum_{Q_{v}=v}^{Q} \sum_{\mathbb{D}_{Q_{v}}^{k}}\left\{\prod_{i=1}^{Q}\left[p_{i}^{k\left(1-I_{k}(i)-J_{k}(i)\right)}\left(1-I_{k}(i) p_{i}^{k-1}\right) p_{i}^{(k-1) J_{k}(i)}\left(1-J_{k}(i) p_{i}\right)\right] q_{G\left(D_{Q_{v}}^{k}\right)}^{l-1}\left(1-q_{G\left(D_{Q_{v}}^{k}\right)}\right)\right\} \\
& P\left(E_{L, 1}^{\prime}\right)=\sum_{Q_{v}=1}^{v-1} \sum_{\mathbb{D}_{Q_{v}}^{k}}\left\{\prod_{i=1}^{Q}\left[p_{i}^{L_{\max }\left(1-I_{L_{\max }}(i)-J_{L_{\max }}(i)\right)}\left(1-I_{L_{\max }}(i) p_{i}^{L_{\max }-1}\right) p_{i}^{\left(L_{\max }-1\right) J_{L_{\max }}(i)}\left(1-J_{L_{\max }}(i) p_{i}\right)\right]\left(1-q_{G\left(D_{Q_{v}}^{k}\right)}\right)\right\} .
\end{aligned}
$$

Moreover,

$$
\begin{aligned}
\mathbb{D}_{Q_{v}}^{k}=\left\{\left[\left(I_{k}(1), I_{k}(2), \ldots, I_{k}(Q)\right),\left(J_{k}(1), J_{k}(2), \ldots, J_{k}(Q)\right)\right]:\right. \\
\\
\left.\sum_{i=1}^{Q} I_{k}(i)<v, \sum_{i=1}^{Q} I_{k}(i)+\sum_{i=1}^{Q} J_{k}(i)=Q_{v}\right\}
\end{aligned}
$$

is defined as the set that $Q_{v}$ RSs receive the packet correctly within $k$ transmissions and $G\left(D_{Q_{v}}^{k}\right)$ is defined as the indexes of the RSs with $I_{k}(i)+J_{k}(i) \geq 1$ corresponding to each element of $\mathbb{D}_{Q_{v}}^{k}$.

Outage probability is defined as the probability that a packet is lost, which can be expressed as

$$
\begin{aligned}
& P=P\left(E_{1}^{c} \bigcup E_{2}^{c} \bigcup \ldots \bigcup E_{L_{\max }}^{c} \bigcup E_{\emptyset} \bigcup E_{L, 1}^{\prime c}\right) \\
& =\sum_{k=1}^{L_{\max }} P\left(E_{k}^{c}\right)+P\left(E_{L, 1}^{c}\right)+P\left(E_{\emptyset}\right),
\end{aligned}
$$

where the probability that $E_{\emptyset}$ occurs is given by

$$
P\left(E_{\emptyset}\right)=p_{1}^{L_{\max }} p_{2}^{L_{\max } \ldots p_{Q}^{L_{\max }},}
$$

and the probabilities that $E_{k}^{c}$ and $E_{L, 1}^{\prime c}$ occur are given in (3) and (4), respectively.

\section{B. Delay Analysis}

Delay of a packet is defined as the total number of transmissions required to transmit this packet, i.e., $L(n)$. The average delay of a packet can be expressed by

$$
\begin{aligned}
\bar{T} & =\sum_{k=1}^{L_{\max }} \sum_{l=1}^{L_{\max }-k+1}(k+l-1) P\left(E_{k, l}\right) \\
& +L_{\max } P\left(E_{L, 1}^{\prime}\right)+L_{\max } P,
\end{aligned}
$$

and the average delay of a successfully received packet can be expressed by
$\bar{T}=\frac{\sum_{k=1}^{L_{\max }} \sum_{l=1}^{L_{\max }-k+1}(k+l-1) P\left(E_{k, l}\right)+L_{\max } P\left(E_{L, 1}^{\prime}\right)}{\sum_{k=1}^{L_{\max }} \sum_{l=1}^{L_{\max }-k+1} P\left(E_{k, l}\right)+P\left(E_{L, 1}^{\prime}\right)}$.

where $P$ is the outage probability defined in (10) while $P\left(E_{k, l}\right)$ and $P\left(E_{L, 1}^{\prime}\right)$ are given in (5) and (6), respectively.

\section{Throughput Analysis}

Throughput is defined as the average number of correctly received packets per transmission. In the proposed scheme, the first TS and fourth TS of the same transmission could transmit different packets, which make the throughput analysis very complicated. Thus, in this paper, we assume that the BS will only transmit a new packet to RSs after the current packet is correctly received by the MS or when $L(n)=L_{\max }$. Therefore, the throughput can be expressed by

$$
S=\frac{1-P}{\bar{T}},
$$

where $P$ and $\bar{T}$ are computed from (10) and (12), respectively.

\section{Case Study: Two RSs}

In this subsection, we consider a case study for two RSs, i.e., $Q=2$. The impact of predefined threshold $v$ is demonstrated with $v=1$ (Scheme A) and $v=2$ (Scheme B).

1) Throughput Analysis for Scheme A: From (11), $P\left(E_{\emptyset}\right)$ can be expressed as

$$
P\left(E_{\emptyset}\right)=p_{1}^{L_{\max }} p_{2}^{L_{\max }} .
$$

Since $\mathbb{D}_{1}^{k}=\{[(0,0),(1,0)],[(0,0),(0,1)]\}$, and $\mathbb{D}_{2}^{k}=$ $\{[(0,0),(1,1)]\}, P\left(E_{k}^{c}\right)$ given in (3) becomes 


$$
\begin{aligned}
& P\left(E_{k}^{c}\right)=p_{1}^{k-1} p_{2}^{k-1}\left(1-p_{1}\right)\left(1-p_{2}\right) q_{12}^{L_{\max }+1-k}+ \\
& p_{2}^{k} p_{1}^{k-1}\left(1-p_{1}\right) q_{1}^{L_{\max }+1-k}+p_{1} p_{2}^{k-1}\left(1-p_{2}\right) q_{2}^{L_{\max }+1-k}
\end{aligned}
$$

and $P\left(E_{L, 1}^{\prime c}\right)$ given in (4) can be simplified to

$$
P\left(E_{L, 1}^{\prime c}\right)=0 \text {. }
$$

Substituting (15), (16) and (17) into (10), we can obtain the outage probability for Scheme A given by

$$
\begin{aligned}
& P=\left(1-p_{2}\right) p_{1} q_{2} \frac{q_{2}^{L_{\max }}-p_{2}^{L_{\max }} p_{1}^{L_{\max }}}{q_{2}-p_{1} p_{2}} \\
& +\left(1-p_{1}\right) p_{2} q_{1} \frac{q_{1}^{L_{\max }}-p_{2}^{L_{\max }} p_{1}^{L_{\max }}}{q_{1}-p_{1} p_{2}} \\
& +\left(1-p_{1}\right)\left(1-p_{2}\right) q_{12} \frac{q_{12}^{L_{\max }}-p_{1}^{L_{\max }} p_{2}^{L_{\max }}}{q_{12}-p_{1} p_{2}}+p_{1}^{L_{\max }} p_{2}^{L_{\max }} .
\end{aligned}
$$

From (5), $P\left(E_{k, l}\right)$ can be expressed as

$$
\begin{aligned}
& P\left(E_{k, l}\right)=p_{1}^{k-1} p_{2}^{k-1}\left(1-p_{1}\right)\left(1-p_{2}\right) q_{12}^{l-1}\left(1-q_{12}\right) \\
& +p_{1}^{k-1} p_{2}^{k}\left(1-p_{1}\right) q_{1}^{l-1}\left(1-q_{1}\right)+p_{1}^{k} p_{2}^{k-1}\left(1-p_{2}\right) q_{2}^{l-1}\left(1-q_{2}\right),
\end{aligned}
$$

and $P\left(E_{L, 1}^{\prime}\right)$ given in (6) can be simplified to

$$
P\left(E_{L, 1}^{\prime}\right)=0 \text {. }
$$

The delay can be obtained by substituting (18), (19) and (20) into (12), and the throughput can be further obtained from (14).

2) Throughput Analysis for Scheme B: From (11), $P\left(E_{\emptyset}\right)$ can be expressed as

$$
P\left(E_{\emptyset}\right)=p_{1}^{L_{\max }} p_{2}^{L_{\max }}
$$

Since

$$
\begin{aligned}
& \mathbb{D}_{1}^{k}=\{[(0,0),(1,0)],[(0,0),(0,1)],[(1,0),(0,0)],[(0,1),(0,0)]\}, \\
& \mathbb{D}_{2}^{k}=\{[(0,0),(1,1)],[(0,1),(1,0)],[(1,0),(0,1)]\}, P\left(E_{k}^{c}\right) \\
& \text { becomes } \\
& \begin{aligned}
P\left(E_{k}^{c}\right) & =\left[\left(1-p_{1}^{k-1}\right) p_{2}^{k-1}\left(1-p_{2}\right)+\left(1-p_{2}^{k-1}\right) p_{1}^{k-1}\left(1-p_{1}\right)\right. \\
& \left.+p_{1}^{k-1} p_{2}^{k-1}\left(1-p_{1}\right)\left(1-p_{2}\right)\right] q_{12}^{L_{\max }+1-k}
\end{aligned}
\end{aligned}
$$

and $P\left(E_{L, 1}^{\prime c}\right)$ is simplified to

$$
P\left(E_{L, 1}^{\prime c}\right)=p_{1}^{L_{\max }}\left(1-p_{2}^{L_{\max }}\right) q_{2}+p_{2}^{L_{\max }}\left(1-p_{1}^{L_{\max }}\right) q_{1} .
$$

Substituting (21), (22) and (23) into (10), the outage probability for Scheme B is given by

$$
\begin{aligned}
& P=\left(1-p_{2}\right) \frac{q_{12}^{L_{\max }+1}-p_{2}^{L_{\max }} q_{12}}{q_{12}-p_{2}} \\
& +\left(1-p_{1}\right) \frac{q_{12}^{L_{\max }+1}-p_{1}^{L_{\max }} q_{12}}{q_{12}-p_{1}} \\
& +\left(p_{1} p_{2}-1\right) \frac{q_{12}^{L_{\max }+1}-p_{1}^{L_{\max }} p_{2}^{L_{\max }} q_{12}}{q_{12}-p_{1} p_{2}} \\
& +p_{1}^{L_{\max }} p_{2}^{L_{\max }}+p_{1}^{L_{\max }}\left(1-p_{2}^{L_{\max }}\right) q_{2}+p_{2}^{L_{\max }}\left(1-p_{1}^{L_{\max }}\right) q_{1} .
\end{aligned}
$$

From (5), $P\left(E_{k, l}\right)$ can be expressed as

$$
\begin{aligned}
& P\left(E_{k, l}\right)=\left[\left(1-p_{1}^{k-1}\right) p_{2}^{k-1}\left(1-p_{2}\right)+\left(1-p_{2}^{k-1}\right) p_{1}^{k-1}\left(1-p_{1}\right)\right. \\
& \left.+p_{1}^{k-1} p_{2}^{k-1}\left(1-p_{1}\right)\left(1-p_{2}\right)\right] q_{12}^{l-1}\left(1-q_{12}\right),
\end{aligned}
$$

and $P\left(E_{L, 1}^{\prime}\right)$ can be simplified to

$P\left(E_{L, 1}^{\prime}\right)=p_{1}^{L_{\max }}\left(1-p_{2}^{L_{\max }}\right)\left(1-q_{2}\right)+p_{2}^{L_{\max }}\left(1-p_{1}^{L_{\max }}\right)\left(1-q_{1}\right)$.

Similar to Scheme A, the delay and throughput can be obtained from (12) and (14), respectively.

\section{SIMULATION RESULTS}

\section{A. Simulation Setup}

We consider the case of two RSs. When both RSs receive the packet correctly, they will cooperatively transmit using the distributed Alamouti space-time code [12]. We assume that

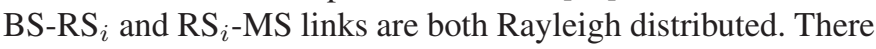
are 40 QPSK symbols in one packet. The channels are kept constant over one packet and vary independently from packet to packet. Only if all the symbols in a packet are decoded correctly, the packet is considered to be received successfully.

In simulations, we also consider two conventional schemes which use single RS as the benchmark:

- Scheme C: If there is only one RS receives the packet correctly, that reliable RS will forward the packet to the MS. When both RSs receive the packet correctly, the best $R S^{2}$ will forward the packet to the MS. If none of RSs receive the packet correctly, the $\mathrm{BS}$ will retransmit the packet to the RSs till at least one of the RSs receives the packet correctly.

- Scheme D: If there is only one RS receives the packet correctly, that reliable RS will forward the packet to the MS. When both RSs receive the packet correctly, a randomly selected $R S$ will forward the packet to the MS. If none of RSs receive the packet correctly, the BS will retransmit the packet to the RSs till at least one of the $\mathrm{RSs}$ receives the packet correctly.

\section{B. Simulation Results}

Fig. 3 shows the PER performance comparison for the second part of transmission to the MS from the following $\mathrm{RS}(\mathrm{s})$ : i) either $R S_{1}$ or $R S_{2}$ (single relay), ii) the best $\mathrm{RS}$ among $R S_{1}$ and $R S_{2}$ (best relay), and iii) both $R S_{1}$ and $R S_{2}$ (cooperative relay). It can be seen that the cooperative relay case achieves the best performance compared to the best relay and the single relay cases. For example, at $P E R=10^{-2}$, the cooperative relay case is approximately $2 \mathrm{~dB}$ and $12 \mathrm{~dB}$ better than the best relay and the single relay cases, respectively.

Figs. 4-8 compare the throughput, outage probability and delay performance for different schemes. Since the quality of the BS-RS ${ }_{i}$ links are usually good, we consider the signal-toratios (SNRs) of $\mathrm{BS}-\mathrm{RS}_{i}$ links $\left(S N R_{1}\right)$ fixed at $16 \mathrm{~dB}$ and

${ }^{2}$ Here, we assume that the best relay is selected among $\mathrm{RS}_{1}$ and $\mathrm{RS}_{2}$ according to the channel power gains of $\mathrm{RS}_{i}$-MS links in each packet. 


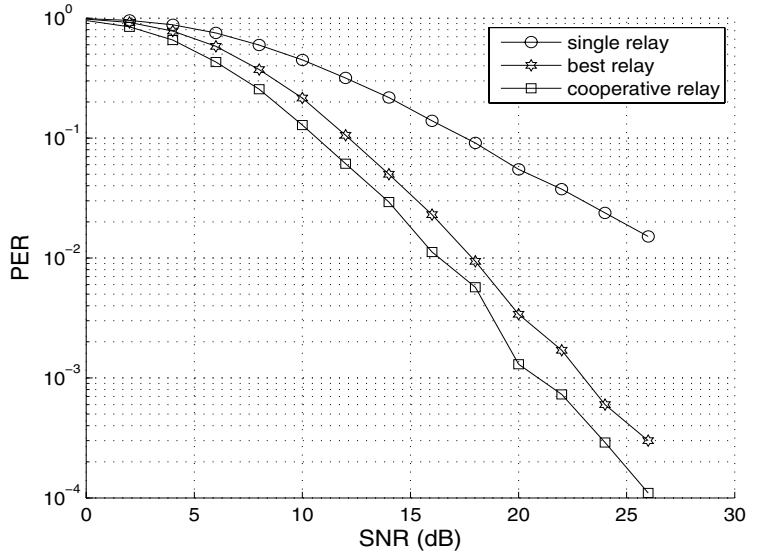

Fig. 3. PER comparison for single relay, best relay and cooperative relay transmission schemes.

$26 \mathrm{~dB}$. As can be seen from these figures, all the theoretical curves match perfectly with our simulation results. Fig. 4 shows the outage probability of Scheme A and Scheme B for different $L_{\max }$ and different $\mathrm{SNR}$ of $\mathrm{RS}_{i}$-MS links $\left(S N R_{2}\right)$ with $S N R_{1}$ fixed at $16 \mathrm{~dB}$. As can be seen, the outage probability reduces significantly when $L_{\max }$ is increased from 4 to 20 . For a fixed $L_{\max }$, the outage probabilities of both schemes decrease with the increasing of $S N R_{2}$. However, at high $S N R_{2}$, the outage probability performance is dominated by the BS-RS links which explains why Scheme B has slightly worse outage performance than Scheme A.

Fig. 5 illustrates that the average delay of Scheme A and Scheme B increase significantly with the increase of $L_{\max }$ when $S N R_{2}$ is small and keep unchanged when $S N R_{2}$ is medium and high. This is mainly due to the fact that the number of retransmissions for the second part will be increased when the RSs-MS links are poor while it does not vary much when the RSs-MS links are good.

Figs. 6 and 7 show the throughput comparison of Schemes A, B, C, D with $L_{\max }=4$ for different $S N R_{1}$ and $S N R_{2}$. By fixing $S N R_{1}=16 \mathrm{~dB}$ (see Fig. 6), the throughput of Scheme A outperforms Scheme B for large $S N R_{2}$ values (i.e., $S N R_{2}>7.5 \mathrm{~dB}$ ) while it slightly underperforms Scheme B for small $S N R_{2}$ values (i.e., $S N R_{2}<7.5 \mathrm{~dB}$ ). This is mainly due to the fact that when $S N R_{2}$ is large (i.e., the $\mathrm{RS}_{i}$-MS links are good), it is better to set a smaller threshold value (i.e., Scheme A) since the benefit of cooperative diversity brought by a larger threshold value can not compensate for the longer delay in the first part. However, when $S N R_{2}$ is small, the delay in the second part becomes severe and it is better to set a larger threshold (i.e., Scheme B) in order to reduce the delay by exploring the benefit of cooperative diversity.

In Fig. 7, as the $S N R_{1}$ value is increased to $26 \mathrm{~dB}$, the throughput difference between Scheme A and Scheme $\mathrm{B}$ is reduced. This is due to the fact that when ${\mathrm{BS}-\mathrm{RS}_{i}}_{i}$ links are good, the probability that both RSs will receive the packet correctly is very high. We notice that the throughput

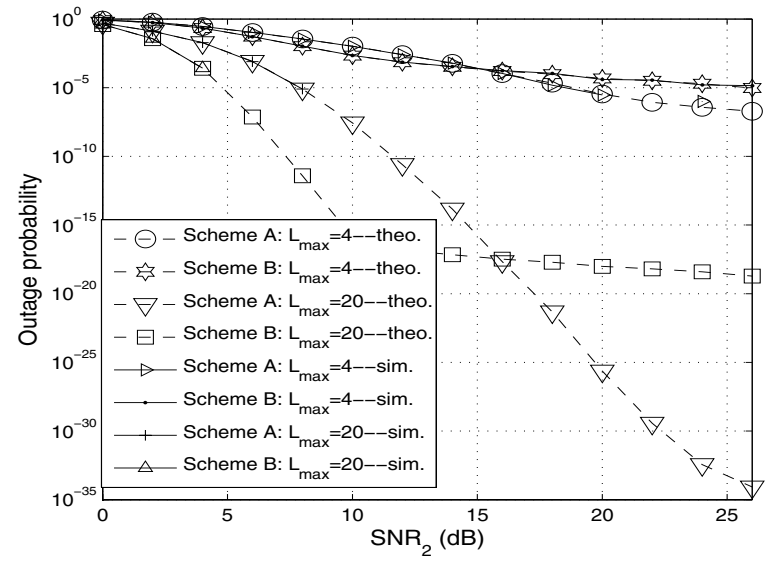

Fig. 4. Outage probability comparison under different $L_{\max }$ for $S N R_{1}=$ $16 \mathrm{~dB}$. (Due to the limitation of the simulation running time, only the outage probabilities smaller than $10^{-5}$ are shown in the figure.)

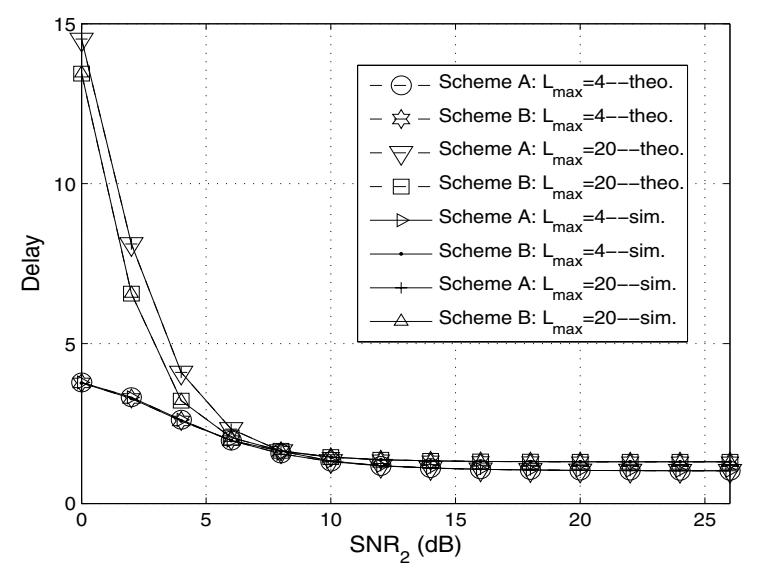

Fig. 5. Delay comparison under different $L_{\max }$ for $S N R_{1}=16 \mathrm{~dB}$.

performance of both schemes are almost identical for small $S N R_{2}$ values (i.e., $S N R_{2}<10 d B$ ) with Scheme A slightly outperforming Scheme B at high $S N R_{2}$ value while the outage probability of Scheme B is smaller than that of Scheme A as shown in Fig. 8. Thus, when BS-RSs links are very good (e.g., $S N R_{1}=26 d B$ ), it is better to set a higher threshold (i.e., Scheme B) in order to reduce the outage probability with a slight degradation in the throughput. As a benchmark, the curves of Scheme C and Scheme D are also included. It is clear that the performance of Scheme A is superior over the single relay case (both Scheme C and Scheme D) due to the cooperative diversity gain.

\section{CONCLUSIONS}

In this paper, an opportunistic cooperative ARQ transmission scheme is proposed. Based on this scheme, two new types of messages, namely, R-ACK/R-NACK, i.e., feedback message from the RSs in a multicast group, and C-ACK/C-NACK, i.e., feedback message from MS to all RSs are introduced. 


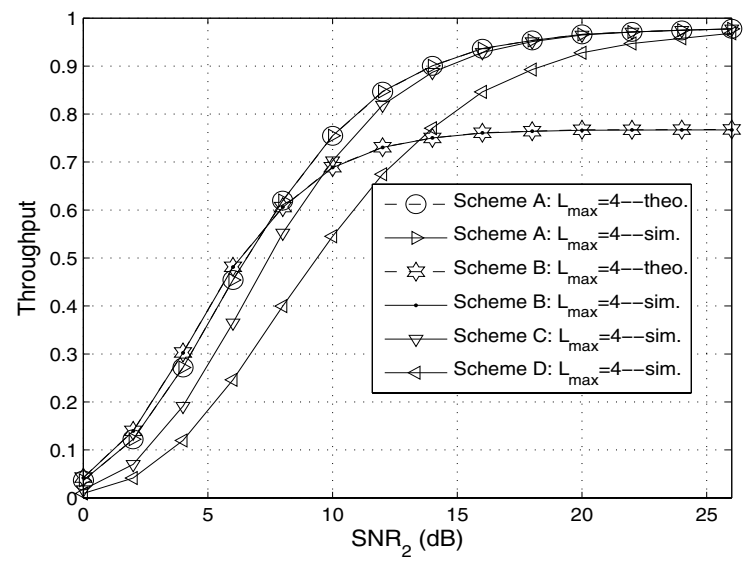

Fig. 6. Throughput comparison for $S N R_{1}=16 \mathrm{~dB}$.

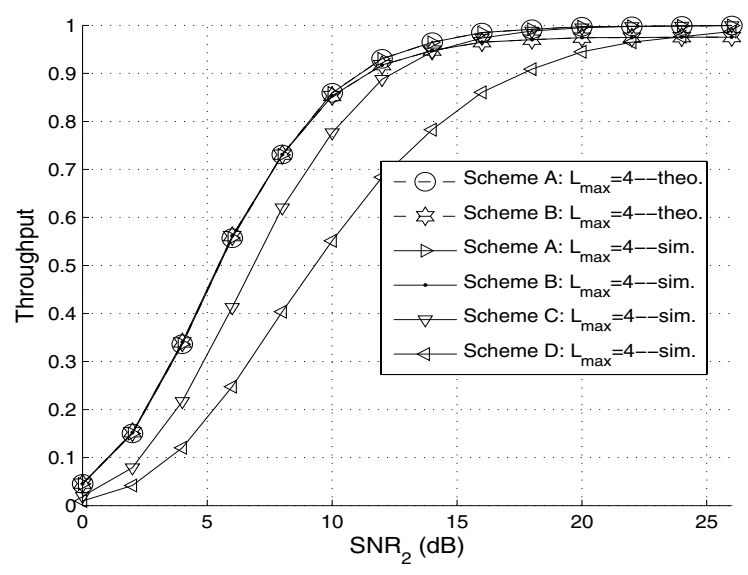

Fig. 7. Throughput comparison for $S N R_{1}=26 \mathrm{~dB}$.

Note that these ACK/NACK messages are used for multicast transmission, which are different from the conventional ACK/NACK used in the unicast transmission only. The BS retransmits a packet to RSs until the number of reliable RSs is larger than a pre-defined threshold. When this is fulfilled, the transmission between BS and RSs is considered complete and the reliable RSs will transmit packets to the MS in a cooperative manner. This clearly shows that the proposed scheme can reduce the burden of the BS. The impact of different thresholds is studied with the outage probability, delay and throughput analysis, which shows that when BS$\mathrm{RS}_{i}$ links are good and if the $\mathrm{RS}_{i}$-MS links are poor, it is more reliable to set a higher threshold value and if the $\mathrm{RS}_{i}$ MSs links are good, it is sufficient to set a low threshold

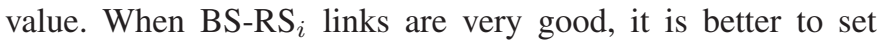
a higher threshold value to achieve lower outage probability. The performance analysis and simulation results clearly show that our proposed scheme can achieve better performance.

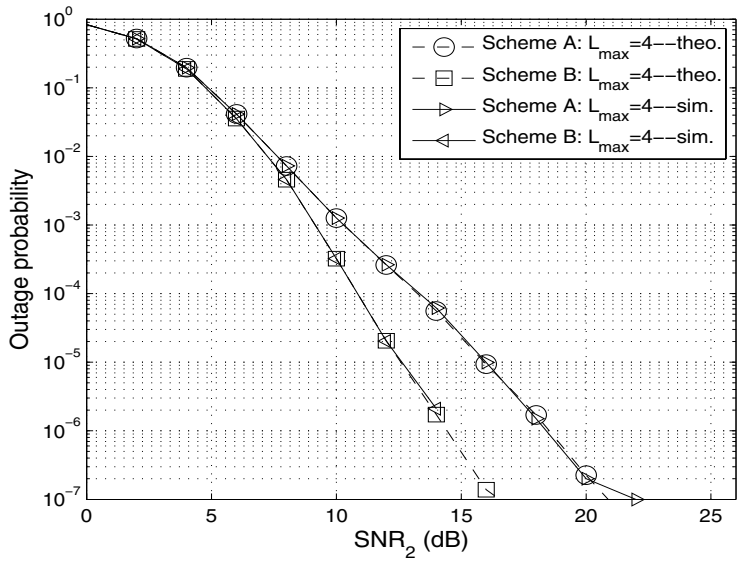

Fig. 8. Outage probability comparison for $S N R_{1}=26 \mathrm{~dB}$.

\section{REFERENCES}

[1] A. Sendonaris, E. Erkip, and B. Aazhang, "User cooperation diversitypart I: System description," IEEE Trans. Commun., vol. 51, pp. 19271938, Nov. 2003.

[2] A. Sendonaris, E. Erkip, and B. Aazhang, "User cooperation diversitypart II: Implementation aspects and performance analysis," IEEE Trans. Commun., vol. 51, pp. 1939-1948, Nov. 2003.

[3] A. Nosratinia, T.E. Hunter, and A. Hedayat, "Cooperative communication in wireless networks," IEEE Communications Magazine, vol. 42 , no. 10, pp. 68-73, 2004.

[4] J. N. Laneman and G. W. Wornell, "Distributed space-time-coded protocols for exploiting cooperative diversity in wireless networks," IEEE Trans. Inf. Theory, vol. 49, no. 10, pp. 2415-2425, Otc. 2003.

[5] J. N. Laneman, D. N. C. Tse, and G. W. Wornell, "Cooperative diversity in wireless networks: Efficient protocols and outage behavior," IEEE Trans. Inf. Theory, vol. 51, no. 12, pp. 3062-3080, Dec. 2004.

[6] Q. Liu, S. Zhou, and G. B. Giannakis, "Cross-layer combining of adaptive modulation and coding with truncated ARQ over wireless links," IEEE Trans. Wireless Comm., vol. 3, pp. 1746-1755, Sep. 2004.

[7] B. Zhao and M. C. Valenti, "Practical relay networks: A generalization of hybrid-ARQ," IEEE J. Sel. Areas Commun., vol. 23, no. 1, pp. 7-18, Jan. 2005

[8] C. K. Lo, Jr. R. W. Heath, and S. Vishwanath, "Hybrid-ARQ in multihop networks with opportunistic relay selection," in IEEE Int. Conf. on Acoustics, Speech, and Signal Proc., Honolulu, Hawaii, Apr. 15-20 2007, vol. 3, pp. 617-620.

[9] T. Tabet, S. Dusad, and R. Knopp, "Achievable diversity-multiplexingdelay tradeoff in half-duplex ARQ relay channels," in IEEE Int. Sym. On Inf. Theory, Sep. 2005, pp. 1828-1832.

[10] G. Yu, Z. Zhang, and P. Qiu, "Cooperative ARQ in wireless networks: Protocols description and performance analysis," in IEEE Int. Conf. on Comm., June 2006, vol. 8, pp. 3608-3614.

[11] L. Dai and K.B. Letaief, "Cross-layer design for combining cooperative diversity with truncated arq in ad-hoc wireless networks," in IEEE Global Telecomm. Conf., 2005, vol. 6.

[12] S. M. Alamouti, "A simple transmit diversity technique for wireless communications," IEEE J. Sel. Areas Commun., vol. 16, no. 8, pp. 1451-1458, Otc. 1998. 